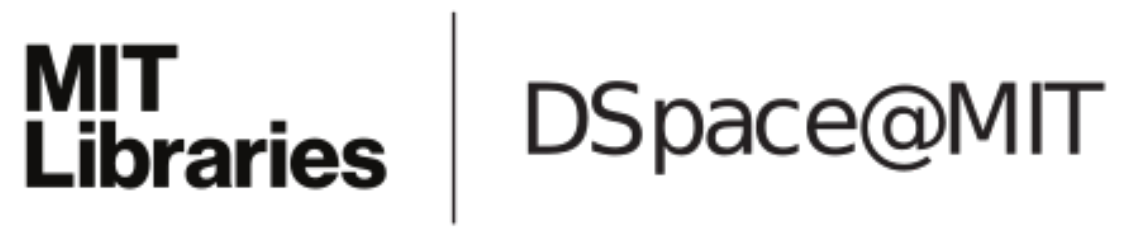

MIT Open Access Articles

Capillary origami in nature

The MIT Faculty has made this article openly available. Please share how this access benefits you. Your story matters.

Citation: Jung, Sunghwan et al. “Capillary origami in nature." Physics of Fluids 21 (2009): 091110.

As Published: http://dx.doi.org/10.1063/1.3205918

Publisher: American Institute of Physics

Persistent URL: http://hdl.handle.net/1721.1/66217

Version: Final published version: final published article, as it appeared in a journal, conference proceedings, or other formally published context

Terms of Use: Article is made available in accordance with the publisher's policy and may be subject to US copyright law. Please refer to the publisher's site for terms of use. 


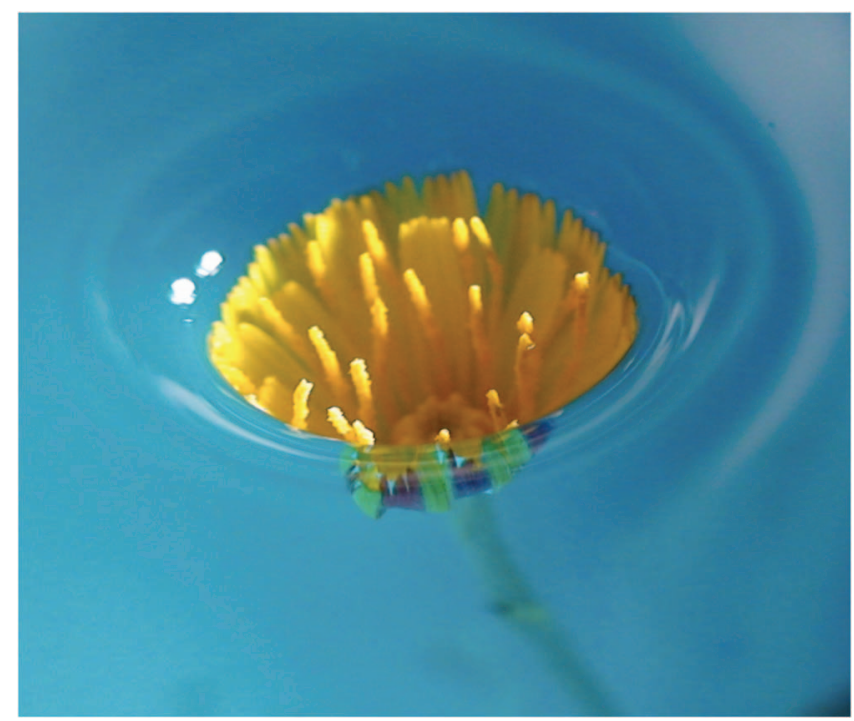

FIG. 1. (Color)

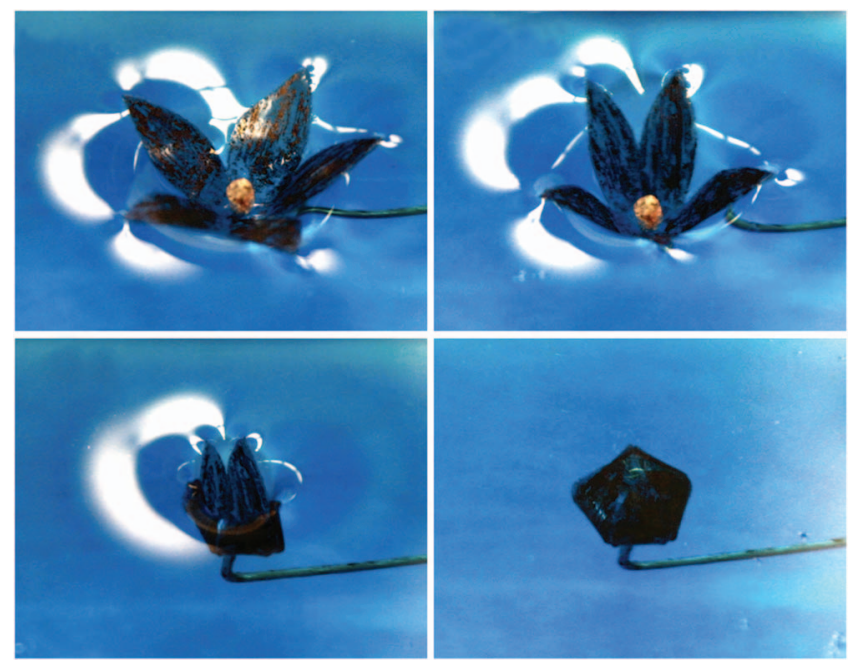

FIG. 2. (Color)

\section{Capillary origami in nature}

Sunghwan Jung, ${ }^{1}$ Pedro M. Reis, ${ }^{1}$ Jillian James, ${ }^{1}$ Christophe Clanet, ${ }^{2}$ and John W. M. Bush ${ }^{1}$

${ }^{1}$ Department of Mathematics, Massachusetts Institute of Technology, Cambridge, Massachusetts 02139, USA

${ }^{2}$ LadHyX, Ecole Polytechnique, Palaiseau 91128, France

(Received 20 July 2009; published online 11 September 2009)

[doi:10.1063/1.3205918]

Capillary forces dominate gravity on a small scale and may deform flexible bodies in both natural and laboratory settings. ${ }^{1}$ Two examples are considered here: floating flowers and spider webs.

Some flowers float on the water surface with their weight supported and shape determined by the combined influence of elastic, capillary, and hydrostatic forces (Fig. 1). Analogous artificial flowers cut out of a flexible polymer sheet similarly deform when submerged (Fig. 2). In the extreme case of complete submergence, the flower may close

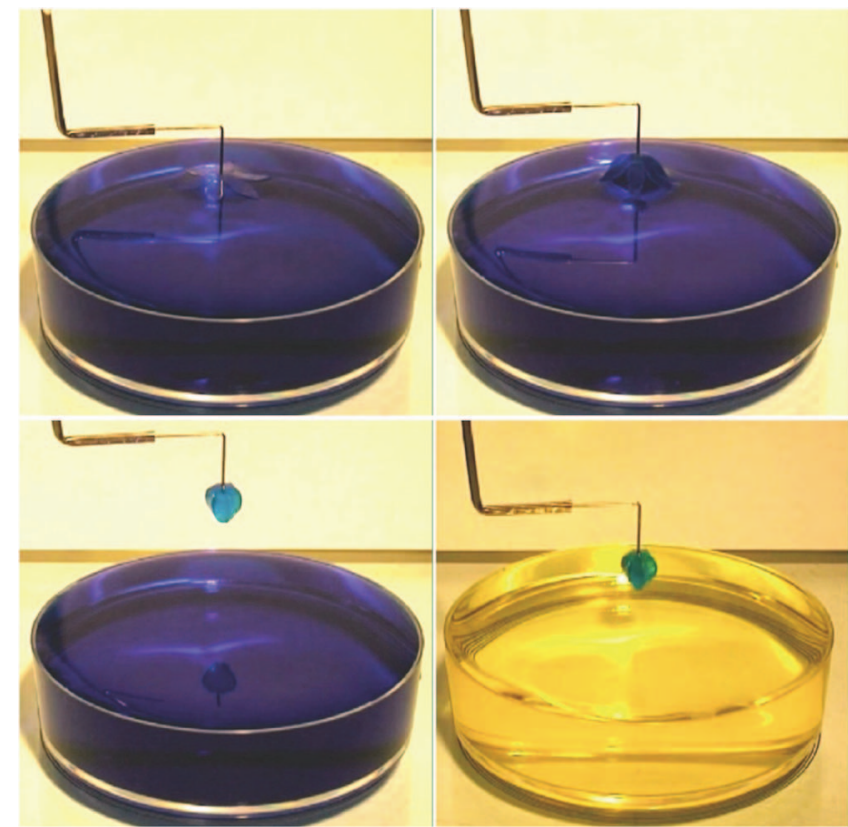

FIG. 3. (Color)

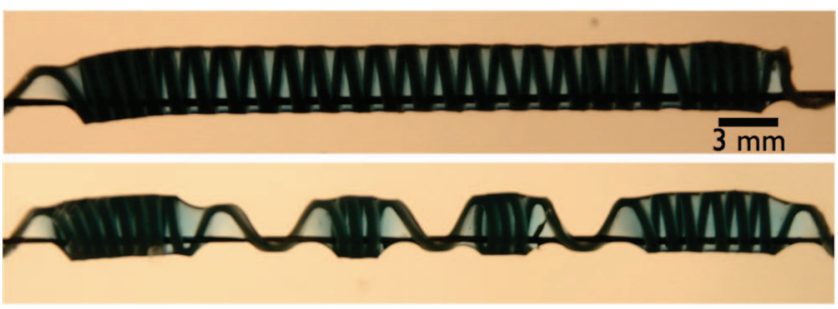

FIG. 4. (Color)

completely, staying dry inside by trapping an enclosed air bubble. When the experiment is inverted, and the artificial flower is withdrawn from the water surface, it instead traps a water droplet of fixed volume, and so serves as an elastocapillary pipette (Fig. 3).

The spider capture threads that run circumferentially around spider webs are typically coated with a viscous fluid. Capillary instability of this film prompts its evolution into a series of fluid droplets, inside of which the slack elastic thread wraps into a series of coils. The result is a characteristic windlass mechanism: ${ }^{2}$ When the prey strikes the web, the coil unravels within the drop, and the associated viscous damping prevents the prey from being ejected. ${ }^{3}$ Analogous laboratory experiments mimic the instability of the spider web (Fig. 4). The elastocapillary instability of a helical elastic thread immersed in silicone oil results in a wavelength prescribed by the interfacial tension and the spring's initial loading.

${ }^{1}$ C. Py, P. Reverdy, L. Doppler, J. Bico, B. Roman, and C. N. Baroud, "Capillary origami: Spontaneous wrapping of a droplet with an elastic sheet," Phys. Rev. Lett. 98, 156103 (2007).

${ }^{2}$ F. Vollrath and D. T. Edmonds, "Modulation of the mechanical properties of spider silk by coating with water," Nature (London) 340, 305 (1989).

${ }^{3}$ Animal Planet and BBC, "Life in the undergrowth," DVD. BBC (UK) (2005). 